senden von Jahren eine 98-Prozent-Männer-Quote gibt? Die Männer müssten sich fragen, ob sie nicht alle Quoten-Männer sind, weil sich offensichtlich viele nicht vorstellen können, dass an diese Stellen Frauen kommen.

Und dann gibt es noch das klassische Ablenkungsmanöver: Die Quote oder der Mangel an einer Quote sei gar nicht das Problem, sondern das Problem sei die mangelnde Vereinbarkeit von Familie und Beruf. Dass das falsch ist, zeigt sich an der Statistik: Bis zu 30 Prozent der Frauen sind kinderlos. Warum kommen diese Frauen nicht in die Vorstands- und Aufsichtsratsetagen? Zudem haben Frauen in Top-Positionen viel weniger Probleme, ihre Kinder betreuen zu lassen, denn sie haben die finanziellen Mittel dafür, sich unterstützen zu lassen.

Insgesamt zeigt sich also, dass die Bestenauslese nicht funktioniert, sondern nach dem Ähnlichkeitsprinzip rekrutiert wird. Männer finden Männer. Auch bei Neueinstellungen. Bei einem großen Autohersteller erzählte mir vor einem Jahr die Gleichstellungsbeauftragte, dass sie in einer Vorstandssitzung die Kollegen mit der Frage konfrontiert hat: „Warum stellen wir eigentlich immer die Dümmsten ein? “. Der Vorstand blickte höchst irritiert. Sie hat die Statistik - mit den besseren Abschlüssen der weiblichen Absolventen neben die erfolgten Neueinstellungen gelegt und gezeigt, dass im Wesentlichen Männer eingestellt wurden. Eine Provokation, die bestehende Klischees in Frage stellt.

Wir alle wissen: Wenn wir es versäumen, den Weg für die besten Talente frei zu machen, haben wir ein Innovations- und Wettbewerbsproblem. Dann werden wir in die Mittelmäßigkeit abrutschen. Es gibt sogar Wirtschaftsbosse, die das mittlerweile selbst sagen. Ich glaube aber, dass wir sie bei dem Prozess, diese Erkenntnis in die Realität umzusetzen, unterstützen müssen, weil er sonst zu lange dauert.

Jetzt braucht es auch Mut im Deutschen Bundestag. Den wünsche ich mir. Ich will Ramona Pisal zitieren. Sie haben in einem Beitrag beim Deutschlandradio mal gesagt: „Die Straßenverkehrsordnung postuliert, dass die Teilnahme am Straßenverkehr ständige Vorsicht und gegenseitige Rücksicht erfordere. Ampeln, Verkehrszeichen und -regeln, Bußgelder und Strafen verraten, dass die Gesellschaft sich nicht allein auf die Einsicht und das Verantwortungsgefühl der Verkehrsteilnehmer verlassen mag."
Wer also für weitere Selbstverpflichtungen ist, könnte - um im Bild zu bleiben - auch fordern, rote Ampeln abzuschaffen.

Es gilt beim Thema Frauen und Gleichstellung das gleiche wie für die Straßenverkehrsordnung: Wir glauben zwar, dass die Menschen alle gut sind, aber wir vertrauen nicht darauf, dass alle ausreichend rücksichtsvoll fahren. Und genauso, wie wir eben nicht fordern, die roten Ampeln abzuschaffen, weil die Menschen das schon aus einem „Flexi-Verantwortungsgefühl" heraus selber machen, verlassen wir uns 60 Jahre nach dem Grundgesetz auch nicht darauf, dass sich die Gleichstellung von Frauen in Führungspositionen nur mit ein wenig gutem Willen realisieren wird.

Frau Bär hat angesprochen, dass es demnächst ein fraktionsübergreifendes Treffen der Frauen im Bundestag geben soll - organisiert von meiner Kollegin Ekin Deligöz. Doch in meiner Fraktion macht sich eine rasende Ungeduld breit und wir wollen nicht wieder warten und warten. Wir haben unseren Gesetzentwurf für die Aufsichtsräte vor über einem Jahr eingebracht. Er wurde im Ausschuss längst abschließend beraten. Wir wollen mit diesem Antrag noch in diesem Jahr ins Plenum des Deutschen Bundestages ziehen. Wir sind kompromissbereit. Wir Frauen im Deutschen Bundestag müssen unsere Macht jetzt nutzen. Wie bei der Stammzellforschung und der Patientenverfügung auch, geht es bei den Aufsichtsräten um eine Grundsatzfrage.

Über 60 Jahre nach Schaffung des Grundgesetzes ist der Zeitpunkt gekommen, wo die Frauen im Bundestag eine entscheidende Frage zur Herstellung tatsächlicher Gleichstellung in Deutschland zur Grundsatzfrage erklären müssen. Sonst wird aus der Pflichtaufgabe im Grundgesetz, aktiv die Gleichstellung zu fördern, noch die nächsten 50 Jahre keine neue Realität werden. Wenn wir das jetzige Tempo beibehalten, würde es ungefähr 50 Jahre dauern, bis Frauen zu einem angemessenen Anteil in Führungspositionen vertreten sind. Seit der Einführung des Gleichstellungssatzes in das Grundgesetz wären dann 110 Jahre vergangen. Das ist mir für eine führende Industrienation zu lange.

Wir Frauen sollten, bevor die 110 Jahre vorbei sind, als ein Baustein auf dem Weg zu echter Gleichstellung eine Geschlechterquote für die Aufsichtsräte erreichen. Das ist mein Traum für dieses Jahr. Ich danke Ihnen für Ihre Aufmerksamkeit.

\title{
„Lohnfindung“ als Ursache von Entgeltdiskriminierung
}

\section{Dr. Karin Tondorf \\ Wissenschaftlerin und Beraterin, Seddiner See}

Zum diesjährigen Equal Pay Day soll das Thema „Lohnfindung "1 eingehender diskutiert werden. Damit wird der Blick auf Prozesse der Festsetzung oder Aushandlung von Arbeitsentgelten gelenkt - für viele eine Art „black box“, die schwer durchschaubar ist. Zwar ist das Ergebnis sichtbar: Frauen erhal- ten in den meisten Unternehmen und Tarifbereichen im Durchschnitt deutlich niedrigere Arbeitsentgelte als Männer. Doch wie werden diese Unterschiede begründet? Inwieweit sind sie sachlich gerechtfertigt? Es lohnt sich, hier Licht ins Dunkel zu bringen, denn wissenschaftliche Untersuchungen und Gerichts-

\footnotetext{
1 Der Oberbegriff „Lohn“ hat im Zuge der Vereinheitlichung von Lohn und Gehalt an Bedeutung verloren. Für beide Vergütungsformen wird hier der Oberbegriff „Entgelt“ verwendet.
} 


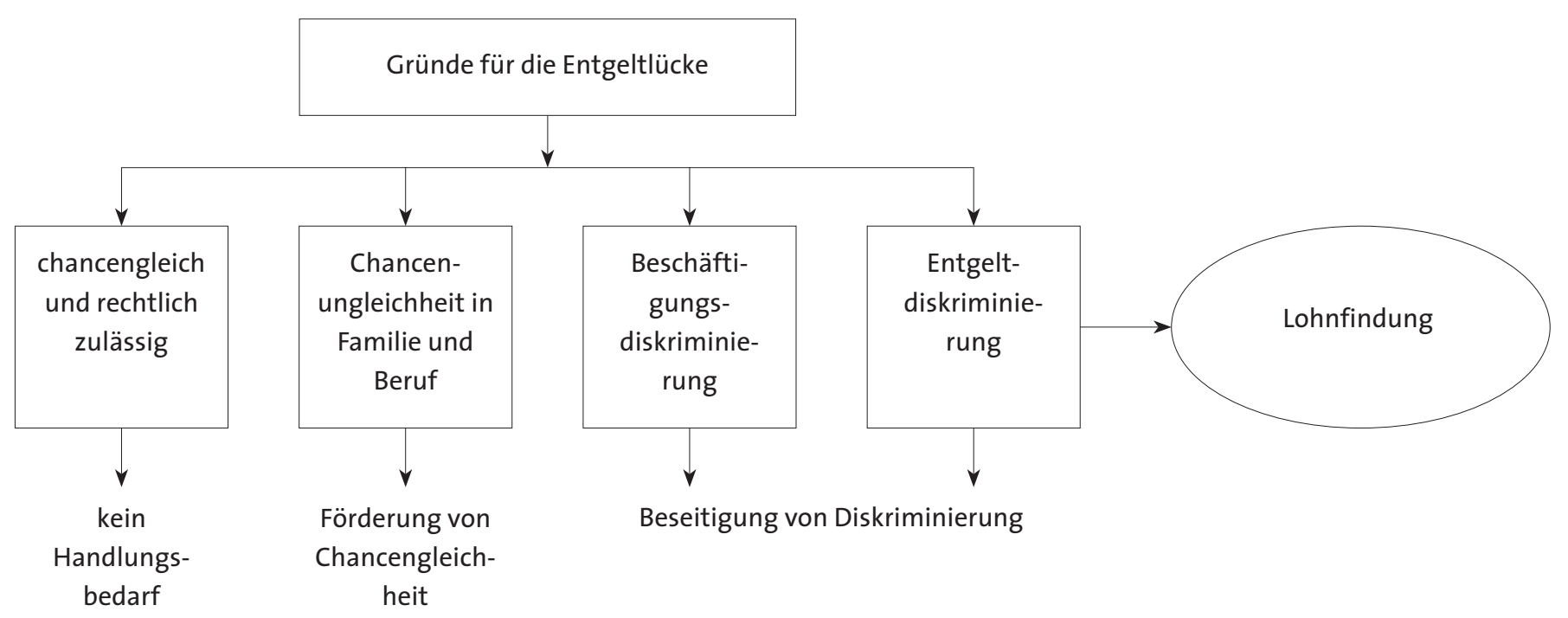

A Abb.1: Gründe für die Entgeltlücke

urteile zeigen, dass es in diesen Prozessen zu Diskriminierungen kommt. ${ }^{2}$ Insofern tragen sie zumindest teilweise dazu bei, dass der Bruttostundenverdienst von Frauen in Deutschland um 23 Prozent geringer ausfällt als der von Männern. Diesen „unbereinigten Gender Pay Gap“ will das Bundesministerium für Familie, Senioren, Frauen und Jugend (BMFSFJ) bis 2020 auf zehn Prozent senken. ${ }^{3}$ Doch so lange die potentiellen Diskriminierungsmechanismen der Lohnfindung nicht aufgedeckt sind, lassen sie sich auch nicht beseitigen.

Zum Thema „Lohnfindung“ sollen drei Fragen geklärt werden: „Was bestimmt die Lohnhöhe? Wer bestimmt über die Lohnhöhe? Was muss sich ändern, um die Lohnlücke zu schließen?"4 Die letztgenannte Frage ist - so die These - zu bescheiden gestellt, doch dies ist an späterer Stelle zu erörtern. Bevor auf diese Fragen eingegangen wird, soll zunächst dargestellt werden, auf welche Ursachen Entgeltunterschiede zwischen den Geschlechtern zurückgeführt werden können und wie die „Lohnfindung“ hier einzuordnen ist.

\section{Lohnfindung als eine Ursache der Entgeltlücke}

Frauen werden aus vielfältigen Gründen schlechter entlohnt als Männer. Hierbei kann grob unterschieden werden zwischen Entgeltdifferenzen, die sachlich begründbar und rechtlich zulässig sind und solchen, die Ergebnis einer Diskriminierung aufgrund des Geschlechts sind. Eine solche Unterscheidung ist hilfreich im Hinblick auf die später zu beantwortende Frage, ob überhaupt gleichstellungspolitischer Handlungsbedarf besteht und falls ja, wer für die Beseitigung von Entgeltunterschieden verantwortlich ist: Sind es die Frauen selbst, ist es der Staat, „die Gesellschaft“ oder sind es einzelne Arbeitgeber/ innen und Tarifvertragsparteien? Diese grobe Einteilung der Gründe für Entgeltunterschiede kann auch Hinweise darauf geben, welches Entgelt Frauen „mit gutem Recht“ einfordern können und welche Forderungen „nur“ ethisch-moralisch begründbar sind. Insoweit geht es auch um die Klärung, was für Arbeitgeber/innen und Tarifparteien rechtliche Pflicht ist und wo die freiwillige Kür beginnt. (s. Abb. 1)

\section{Ursachenkomplexe Entgelt- und Beschäftigungs- \\ diskriminierung}

Entgeltunterschiede zwischen den Geschlechtern können sowohl auf Entgeltdiskriminierung durch Lohnfindung als auch auf Beschäftigungsdiskriminierung beruhen, beispielsweise bei der Stellenausschreibung, Einstellung, Beförderung, Weiterbildung, Umschulung oder bei den Beschäftigungsbedingungen. Ein betriebliches Beispiel: Eine Frau wird bei der Beförderung übergangen, obwohl sie bessere Voraussetzungen hatte als die männlichen Mitbewerber. In beiden Bereichen - Entlohnung und Beschäftigung - ist Diskriminierung aufgrund des Geschlechts verboten, so regelt es $\mathbb{2}$ des Allgemeinen Gleichbehandlungsgesetzes (AGG). Deren Beseitigung ist für die verantwortlichen Arbeitgeber/innen und Tarifparteien gesetzlich verpflichtend, denn es handelt sich hierbei um eine gravierende, die Grundrechte verletzende Form der Benachteiligung. ${ }^{5}$ Deren tiefere Ursachen liegen im Wesentlichen in der herrschenden Geschlechterordnung, die durch sozial konstruierte Unterscheidungen zwischen „Mann“ und „Frau“ (nicht naturgegebene Unterschiede) und einer damit verwobenen Hierarchisierung geprägt ist. ${ }^{6}$ Auch Institutionen, Organisationen und Personen, die für Bestimmung der Beschäftigungsbedin-

2 Vgl. u.a. Krell, Gertraude/Carl, Andrea-Hilla/Krehnke, Anna, Diskriminierungsfreie Bewertung von (Dienstleistungs)Arbeit. Vergleichsprojekt zwischen frauen- und männerdominierten Tätigkeiten in der Stadt Hannover, hrsg. von der Vereinten Dienstleistungsgewerkschaft ver.di, Stuttgart 2001.

3 Vgl. Bundesministerium für Familie, Senioren, Frauen und Jugend (BMFSFJ), 2010 b, Ressortbericht 2010 - Verringerung des Verdienstabstandes zwischen Männern und Frauen, Berlin 2010, S. 3.

4 Vgl. Präsentation des Forum Equal Pay Day <www.equalpayday.de〉 (Zugriff: 8.2.2012), initiiert von Business and Professional Women e.V.

5 Vgl. Art. 3, Abs. 2 u. 3 GG sowie Art. 21 und 23 der Grundrechtecharta der EU.

6 Genauere Ausführungen finden sich u.a. in Knapp, Gudrun-Axeli (2011), Gleichheit, Differenz, Dekonstruktion und Intersektionalität: Vom Nutzen theoretischer Ansätze der Frauen- und Geschlechterforschung für die gleichstellungspolitische Praxis, in Krell, Gertraude/Ortlieb, Renate/Sieben, Barbara (Hrsg.), Chancengleichheit durch Personalpolitik. 6. Auflage, Wiesbaden 2011, S. 71-84. 
gungen sowie die Lohnfindung verantwortlich sind, sind nicht frei von stereotypem, hierarchischem „Schubladendenken“, etwa bei der Frage der Bewertung der Arbeit von Frauen und Männern.

\section{Ursachenkomplex Chancenungleichheit}

Vielfach haben Frauen ungünstigere Ausgangsbedingungen beim Zugang zu Stellen, Führungspositionen, Qualifizierungen, Vollzeitbeschäftigungen oder beim Sammeln von Berufserfahrung. Ein Beispiel: Eine Frau wählt Teilzeitarbeit, weil sie nur so die Kinderbetreuung gewährleisten kann. Dadurch verschlechtert sich indirekt auch ihr Einkommen. Ungleiche Chancen haben ihre tieferen Ursachen ebenfalls in der herrschenden Geschlechterordnung, die sich im Erwerbs- und Familienleben spiegelt, etwa dadurch, dass Frauen noch immer die Hauptverantwortung für Hausarbeit, Kindererziehung und Pflege von Familienangehörigen zugeschrieben und ihre Berufstätigkeit als nachrangig betrachtet wird. Diese Strukturen und stereotypen Denkweisen sind nicht leicht aufzubrechen, denn ihre Aufrechterhaltung verschafft den bislang Begünstigten ökonomische wie machtpolitische Vorteile. Auch Steuerpolitik kann Ungleichheitsstrukturen verfestigten. All diese Einflussfaktoren tragen maßgeblich dazu bei, dass Frauen oftmals weiblich konnotierte Berufe ergreifen, die auf den unteren und mittleren betrieblichen Hierarchiestufen angesiedelt sind, oder ihre Erwerbsarbeit wegen Kinderziehung oder Pflege unterbrechen oder ihre Arbeitszeit reduzieren. Auch bei Einkommenslücken, die Folge von Chancenungleichheit sind, besteht gleichstellungspolitischer Handlungsbedarf: Vielfältige politische, zumeist freiwillige betriebliche oder tarifliche Maßnahmen zielen daher auf die Förderung von Chancengleichheit, insbesondere auf bessere Vereinbarkeit von Familie und Beruf, auf Erhöhung des Anteils von Frauen in männerdominierten Branchen, Berufen oder Führungspositionen sowie auf stärkere Beteiligung von Frauen an Entscheidungsprozessen.

\section{Ursachenkomplex „chancengleich und rechtlich zulässig“}

Entgeltunterschiede können unter Wahrung der Chancengleichheit sachlich gerechtfertigt und rechtlich zulässig sein. Ein Beispiel: Eine Frau wählt Teilzeitarbeit, um ihrem Hobby nachgehen zu können. Allein hier besteht kein Handlungsbedarf.

Bis heute ist nicht bekannt, welcher Anteil am Gender Pay Gap auf Diskriminierung zurückzuführen ist. Zwar wird er mittels Regressionsanalysen in zulässige und nicht zulässige Anteile zerlegt, jedoch enthalten die für zulässig erklärten Entgeltunterschiede bereits Diskriminierung. Bei diesem volkswirtschaftlichen Prüfkonzept spielt das Antidiskriminierungsrecht keine Rolle. ${ }^{7}$ Um das Ausmaß von Entgeltdiskriminierung zu erhellen, müsste die Art und Weise der Lohnfindung auf betrieblicher und tariflicher Ebene näher unter die Lupe genommen werden: Wie wird die Arbeit von Frauen und Männern bewertet? Sind die verwendeten Kriterien und Verfahren der Lohnfindung diskriminierungsfrei? Wird gleiche und gleichwertige Arbeit gleich bezahlt?

\section{Lohnfindung}

\section{Was bestimmt die Lohnhöhe?}

Arbeitsentgelte von Frauen und Männern werden mit Hilfe verschiedener Kriterien begründet. Über die Anwendung dieser Kriterien entscheiden verschiedene Verfahren:

\section{Zu den Verfahren der Lohnfindung}

In kleineren Betrieben wird die Höhe des Arbeitsentgelts oft „frei" verhandelt oder einseitig - mehr oder weniger willkürlich - festgesetzt. Dagegen wird das Entgelt in größeren Unternehmen und Branchen „mit System“ ermittelt. Das heißt: Entgeltunterschiede werden mit Kriterien begründet und es werden spezifische Verfahren genutzt, um die Arbeit, die Leistung oder andere Faktoren zu bewerten. Zur (tariflichen) Bewertung von Arbeit zum Zwecke ihrer Eingruppierung in ein Entgeltgefüge werden in Deutschland überwiegend „summarische“, vereinzelt auch „analytische“ Verfahren der Arbeitsbewertung verwendet. Summarische Verfahren berücksichtigen nur eine überschaubare Anzahl von Bewertungskriterien und bergen daher ein größeres Risiko der Unterbewertung von (Frauen-)Arbeit. Die verwendet Kriterien werden außerdem nicht sichtbar gewichtet. Analytische Verfahren ermöglichen eine differenziertere und transparentere Bewertung, da hierbei jedes Einzelkriterium für sich bewertet und sichtbar gewichtet wird. Allerdings können auch analytische Arbeitsbewertungsverfahren diskriminieren, wenn sie zum Beispiel wesentliche Kriterien an „Frauenarbeitsplätzen“ nicht berücksichtigen. Einen Überblick über Diskriminierungsquellen bei Verfahren der Lohnfindung bietet Abb. 2.

Diskriminierungsquellen bei Verfahren der Lohnfindung

1. „Willkürliche“, unsystematische Setzung von Löhnen.

2. Verfahrensregeln, die große subjektive Spielräume bei ihrer Auslegung eröffnen, z.B. durch „kann“-Vorschriften oder Regelungslücken.

3. Intransparente Bewertungsverfahren, die die Arbeit oder Leistung pauschal bewerten und nicht erkennen lassen, wie die Kriterien gewichtet sind.

4. Uneinheitliche Verfahren, z.B. Stufensteigerungen beim Grundentgelt bei männlichen Arbeitern nach Lebensalter, bei weiblichen Angestellten nach Berufsjahren.

Abb. 2: Diskriminierungsquellen bei Verfahren der Lohnfindung

7 Entgeltgleichheit wird in diesen Analysen anders definiert als nach dem Entgeltgleichheitsrecht: Sie besteht, wenn Frauen und Männer mit gleicher Humankapitalausstattung gleich bezahlt werden. Zur näheren Erläuterung der volkswirtschaftlichen Analysemethode des Gender Pay Gap siehe Finke, Claudia (2011), Verdienstunterschiede zwischen Frauen und Männern. Eine Ursachenanalyse auf Grundlage der Verdienststrukturerhebung 2006, in: Statistisches Bundesamt, Wirtschaft und Statistik, Januar 2011, S. 36-48. 


\section{Zu den Kriterien der Lohnfindung}

Das Bruttoarbeitsentgelt setzt sich in der Regel aus mehreren Bestandteilen zusammen. Sie werden unterschiedlich begründet:

- Das Grundentgelt orientiert sich an den Anforderungen der Arbeitsstelle (Tätigkeit), d.h. nicht nach der Person oder deren Leistung. Häufig verwendete Anforderungskriterien (Tätigkeitsmerkmale) sind „Wissen und Können“, „Verantwortung “, „Selbstständigkeit“.

- Oftmals steigt das Grundentgelt mit den Jahren im Beruf oder in der Tätigkeit. Die einschlägige Berufserfahrung bzw. Tätigkeitsjahre sind dann ein weiteres Differenzierungskriterium.

- Wird eine Leistungszulage oder -prämie gezahlt, stellt die Leistung ein weiteres Kriterium dar.

- Weitere Entgeltbestandteile haben jeweils eigene Kriterien der Entgeltbegründung und -differenzierung, zum Beispiel Schichtarbeit, Erschwernisse, Umsatz, Zielerreichung oder soziale Parameter.

Eine erste Quelle von Entgeltdiskriminierung liegt darin, dass wesentliche Anforderungen der Tätigkeiten von Frauen nicht oder nicht angemessen bewertet und nicht bezahlt werden. Werden in einem Tarifbereich zum Beispiel nur „Wissen und Können“ und „Verantwortung“ bewertet, obwohl bei „Frauentätigkeiten“ auch „Kommunikationsfähigkeit“ oder „Bewegungspräzision“ erforderlich sind, wird die Bezahlung schnell ungerecht. Eine zweite Quelle sind unterschiedliche Kriterien, zum Beispiel bei weiblichen Angestellten und männlichen Arbeitern. Eine solche uneinheitliche Bewertung ist oft in Tarifbereichen zu finden, in denen noch Lohn- und Gehaltstarifverträge existieren. Benachteiligend wirken auch Kriterien, die von Frauen (oder Männern) aufgrund der Arbeitsteilung in der Familie oder der Körperkraft schwerer zu erfüllen sind; dazu gehört das Leistungskriterium der „Flexibilität“. Auch darf nur insoweit nach der „Berufsausbildung“ differenziert werden, als sie für die Ausübung der zu verrichtenden Tätigkeit von Bedeutung ist. ${ }^{8}$ Als gleichheitswidrig wird auch eine Entgeltabsenkung von frauendominierten Tätigkeiten eingeschätzt, die mit dem Argument einer „beschäftigungspolitischen Maßnahme“ erfolgt. ${ }^{9}$ Analysen von Tarifverträgen und Betriebs-/Dienstvereinbarungen haben weitere Probleme bei der Anwendung von Kriterien aufgezeigt. Sie sind in der Abb. 3 aufgelistet.

\section{Wer bestimmt die Lohnhöhe?}

In Deutschland haben einzelne Arbeitgeber/innen und Tarifvertragsparteien maßgeblichen Einfluss auf die Arbeitsentgelte von Frauen und Männern. Auch Betriebs- bzw. Personalrät/ inn/e/n haben Mitbestimmungs- und Mitwirkungsmöglichkeiten, beispielsweise bei der Leistungsvergütung oder bei der Eingruppierung. Für die Besoldung der Beamtinnen und Beamten ist der Gesetzgeber verantwortlich. Damit sind die wesentlichen Akteursgruppen benannt, die Entgeltungleichheit verursachen und zugleich beseitigen können - und müssen. Oftmals sind Frauen an lohnpolitischen Entscheidungen nicht

\section{Diskriminierungsquellen bei Kriterien der Lohnfindung}

1. Kriterienauswahl: Fehlen von Kriterien, die an frauendominierten Arbeitsplätzen auftreten, z.B. Körperkraft bei Pflegepersonal.

2. Verwendung unterschiedlicher Kriterien bei der Bewertung von frauen- und männerdominierten Tätigkeiten.

3. Doppel- oder Mehrfachbewertung von Kriterien, die ähnliche Sachverhalte erfassen und männerdominiert Gruppen begünstigen (z.B. Verantwortung und Selbstständigkeit).

4. Diskriminierende Auslegung von Kriterien oder Verwendung diskriminierungsanfälliger Kriterien, die Frauen oder Männer aus gesellschaftlichen oder physischen Gründen (tradierte familiäre Rollenteilung oder Körperkraft) in unterschiedlichem Maß erfüllen können.

5. Aneinanderbinden von Kriterien, z.B. Bewertung von „besonderer Verantwortung“ nur dann, wenn „gründliche, umfassende Fachkenntnisse" erfüllt sind.

6. Unverhältnismäßig hohe Gewichtung von Kriterien, die vor allem auf männerdominierte Tätigkeiten zutreffen.

$\Delta$ Abb. 3: Diskriminierungsquellen im Bereich der Kriterien der Lohnfindung

angemessen beteiligt - etwa in Verhandlungskommissionen -, so dass sie nur begrenzte Chancen haben, auf eine geschlechtsneutrale Lohnfindung hinzuwirken.

\section{Handlungsmöglichkeiten}

Was muss sich ändern, um die Lohnlücke zu schließen? Diese Frage ist zu bescheiden gestellt. Denn selbst dort, wo die Lohnlücke bereits geschlossen ist, das heißt null Prozent beträgt, kann Entgeltdiskriminierung fortbestehen. Dies lässt sich am Beispiel des ostdeutschen öffentlichen Dienstes, für den keine Verdienstdifferenz festgestellt wurde ${ }^{10}$, verdeutlichen: Dieser Wert wird damit erklärt, dass in Ostdeutschland bessere strukturelle Möglichkeiten der Vereinbarkeit von Familie und Beruf bestehen, familienbedingte Erwerbsunterbrechungen relativ kurz ausfallen und eine allgemein stärker ausgeprägte Erwerbsorientierung insbesondere von Müttern besteht. ${ }^{11}$ Inwieweit Entgeltdiskriminierung durch Lohnfindung existiert, wurde noch nicht analysiert. Jedoch weisen die Tarifverträge des öffentlichen Dienstes - West wie Ost - auf eine diskriminierungsanfällige Arbeitsbewertung hin, so dass Tätigkeiten

8 EuGH v. 17.10.1989, Rs. C-109/88, Danfoss.

9 Vgl. Winter, Regine, Die Regelung der Arbeits- und Wirtschaftsbedingungen durch Tarifnormen, in: Däubler, Wolfgang (Hrsg.), Tarifvertragsgesetz, 2. Auflage, Baden-Baden 2006, S. 514.

10 BMFSFJ, Der Verdienstunterschied von Frauen und Männern im öffentlichen Bereich und in der Privatwirtschaft, Berlin 2009, S. 14. 11 Ebd. 
von Frauen unterbewertet sein könnten. ${ }^{12}$ Träfe dies zu und würden sie geschlechtsneutral bewertet und bezahlt, könnte sich dies möglicherweise in einer statistischen Entgeltdifferenz zugunsten der Frauen ausdrücken. Die Schließung der Lohnlücke kann daher nicht das umfassende Gleichstellungsziel sein. Adäquat wäre die Frage „Was muss sich ändern, um chancengleiche und diskriminierungsfreie Entgeltdifferenzen zu erreichen?"

In diesem Beitrag steht die Lohnfindung und damit die Entgeltdiskriminierung im Mittelpunkt. Für diesen Bereich sollen nachfolgend wichtige Handlungsmöglichkeiten aufgelistet werden:

Der erste Schritt bestünde in einer systematischen Prüfung der Lohnfindung, die Arbeitgeber/innen und Tarifparteien verantworten. Sie ist zentrale Voraussetzung für die Beseitigung von Entgeltdiskriminierung. Bislang ist eine solche Prüfung nicht verbindlich vorgeschrieben, sie erfolgt in der Praxis auch nur selten. Mit eg-check.de ${ }^{13}$ steht seit 2010 kostenlos ein Instrumentarium zur Verfügung, das eine solche Prüfung anleitet.

Der zweite Schritt bestünde darin, dass die entgeltpolitisch Verantwortlichen ihre Entgeltregelungen und -praxis diskriminierungsfrei gestalten. Dies heißt insbesondere:

- Die Tätigkeiten von Frauen und Männern werden einheitlich, d.h. mit denselben Kriterien bewertet. ${ }^{14}$

- Die Entgeltsysteme müssen durchschaubar und überprüfbar gestaltet sein. ${ }^{15}$

- Die Kriterien der Arbeitsbewertung müssen an „die Art der in Frage stehenden Tätigkeit“ gebunden sein, d.h. sie müssen das „Wesen“ der tatsächlich zu verrichtenden Arbeit widerspiegeln. Dabei sind auch solche Kriterien zu berücksichtigen, „hinsichtlich derer die weiblichen Arbeitnehmer besonders geeignet sein können. "16 Bei Tätigkeiten mit bzw. am Menschen sind dies zum Beispiel auch Anforde- rungen an Kommunikationsfähigkeit, Kooperationsfähigkeit und Einfühlungsvermögen.

- Diskriminierungsanfällige Kriterien sollten gestrichen werden (s.o.). Nicht zulässig ist es zum Beispiel, die Entlohnung nach Alter, Erwerbsjahren, persönlichen Ausbildungszeiten $^{17}$, vermuteter Leistung oder Verhandlungsgeschick in der Bewerbungssituation zu differenzieren.

Frauen sollten außerdem gleichberechtigt an den Entscheidungsprozessen - etwa in Tarif- und Verhandlungskommissionen - beteiligt werden. Eine diskriminierungsfreie Bezahlung wird nicht mit dem Freiwilligkeitsprinzip zu erreichen sein. Schon die notwendige Prüfung scheitert vielfach an der Überlegung, dass ein Aufdecken von Entgeltdiskriminierung zusätzliche Personalkosten verursachen könnte. Bislang sehen nur wenige Arbeitgeber/innen die ökonomischen Vorteile, die eine geschlechtergerechte Bezahlung für die Rekrutierung von qualifiziertem Personal und für das Betriebsklima haben kann.

Ein gesetzlicher oder tariflicher Mindestlohn kann - auch wenn er nicht in erster Linie gleichstellungspolitisch begründet wird - die Entgeltsituation vieler Frauen verbessern, indem er das Entgeltniveau der unteren Entgeltgruppen anhebt. Allerdings verspricht der Mindestlohn noch nicht, dass auch die geschlechterbezogenen Ungerechtigkeiten beim Arbeitsentgelt beseitigt werden.

12 Vgl. Beitrag von Krell, Gertraude/Tondorf, Karin, Mittelbare Entgeltdiskriminierung in Tarifverträgen des öffentlichen Dienstes - ein Ende in Sicht?, in: djbZ 4-2011, S. 174-177.

13 www.eg-check.de (entgeltgleichheits-check.de), entwickelt von Karin Tondorf und Andrea Jochmann-Döll, gefördert von der HansBöckler-Stiftung.

14 Vgl. Art. 4 der Richtlinie 2006/54/EG.

15 Vgl. EuGH v. 17.10.1989, Rs. C-109/88, Danfoss.

16 Vgl. EuGH v. 1.7.1986, Rs. C-237/85, Rummler.

17 Vgl. EuGH v. 17.10.1989, Rs. C-109/88, Danfoss.

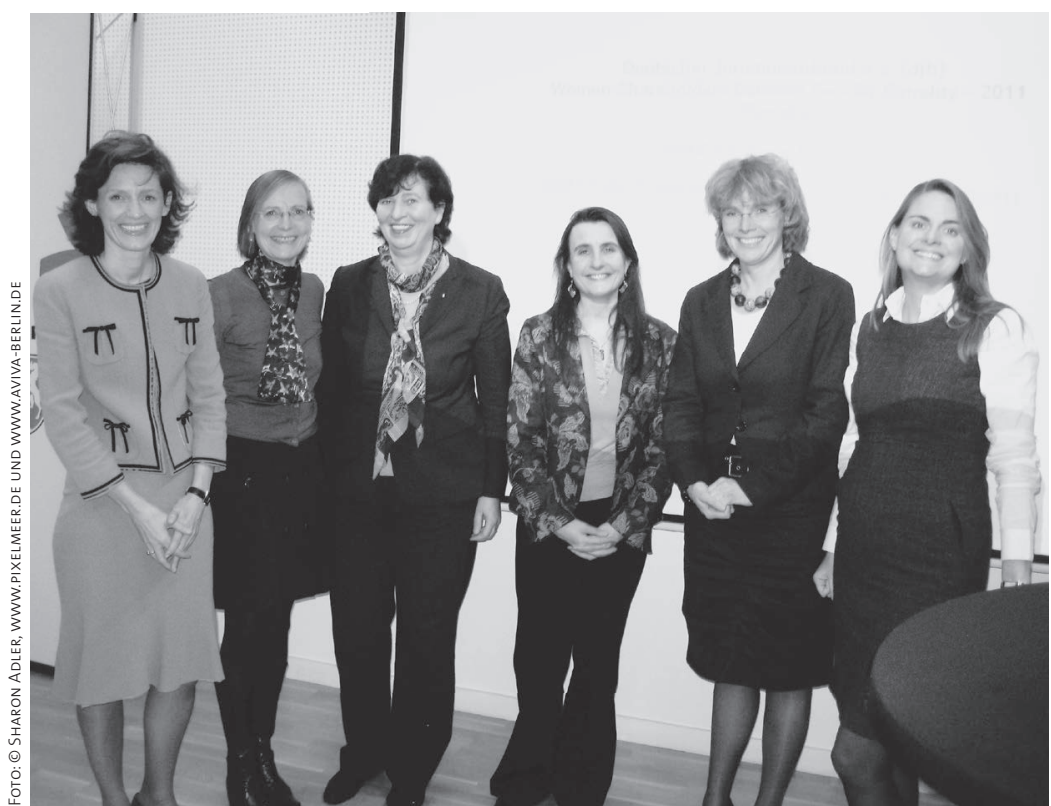

$\varangle$ Mit dem Titel „From words to action - what shall we do to get women at the top?" fand am 23. November 2011 in der Brüsseler Vertretung des Landes Nordrhein-Westfalen eine gemeinsame Veranstaltung der Regionalgruppe Brüssel im djb und des Niederländischen Frauennetzwerkes Brussels NV statt. Margarete Hofmann (Vizepräsidentin des djb) und Henriette van Eijl (Brussels NV) berichteten über die von ihnen beiden am 6. Mai 2011 besuchte Hauptversammlung der Commerzbank AG in Frankfurt. Anschließend stellte Birgit Kersten (Wiss. Mitarbeiterin im Projekt „Aktionärinnen fordern Gleichberechtigung“ des djb) die Studie 2011 vor. Im Anschluss daran gab es eine Podiumsdiskussion mit Anneke Burger (Managerin bei Elektrabel S.A., Co-Autorin des Buches „Ronde Vormen in IT“, Brüssel), Nicole Mönter (The Boston Consulting Group, Brüssel) und Birgit Kersten (Wiss. Mitarbeiterin im Projekt „Aktionärinnen fordern Gleichberechtigung“). Die Gesprächsleitung hatte Sharon Leclercq-Spooner (American Chamber of Commerce EU). V.I.n.r.: Henriette van Eijl, Margarete Hofmann, Birgit Kersten, Sharon Leclercq-Spooner, Anneke Burger, Nicole Mönter. 